\title{
Hiperplasia adrenal congênita em mulheres adultas: manejo de antigos e novos desafios
}

\author{
Congenital adrenal hyperplasia in the adult women: \\ management of old and new challenges
}

Flávia A. Costa-Barbosa' ', Mariana Telles-Silveira' ', Claudio E. Kater ${ }^{1}$

${ }^{1}$ Disciplina de Endocrinologia, Departamento de Medicina, Escola Paulista de Medicina, Universidade Federal de São Paulo (EPM/ Unifesp), São Paulo, SP, Brasil

\author{
Correspondência para: \\ Claudio E. Kater \\ Laboratório de Esteroides, \\ Disciplina de Endocrinologia \\ EPM/Unifesp \\ Rua Pedro de Toledo, 781,13\% andar \\ 04039-032 - São Paulo, SP, Brasil \\ kater@unifesp.br
}

Recebido em 9/Set/2013 Aceito em 25/Nov/2013

\begin{abstract}
RESUMO
Graças ao significativo avanço na conduta e no tratamento de pacientes com as diversas formas de hiperplasia adrenal congênita por deficiência de 21-hidroxilase (D21OH) durante a infância e a adolescência, essas mulheres puderam atingir a idade adulta. Dessa maneira, o manejo nessa fase tornou-se ainda mais complexo, originando novos desafios. Tanto a exposição continuada à corticoterapia (pelo uso de doses muitas vezes suprafisiológicas), quanto ao hiperandrogenismo (pelo tratamento irregular ou uso de doses insuficientes), pode causar resultados pouco favoráveis à saúde e à qualidade de vida dessas mulheres, como: osteoporose, complicações metabólicas com risco cardiovascular, prejuízos cosméticos, infertilidade e alterações psicossociais e psicossexuais. No entanto, há poucos estudos de seguimento de longo prazo nas pacientes adultas. Nessa revisão procuramos abordar alguns aspectos importantes e mesmo controversos no seguimento de mulheres adultas com $\mathrm{D} 210 \mathrm{H}$, recomendando a adoção de terapia individualizada e de caráter multidisciplinar, enquanto novos estudos não proponham atitudes mais bem definidas e consensuais visando à meIhora da qualidade de vida dessas mulheres. Arq Bras Endocrinol Metab. 2014;58(2):124-31
\end{abstract}

\section{Descritores}

Deficiência de 21-hidroxilase; hiperplasia adrenal congênita; tratamento; aspectos psicossociais e psicossexuais

\begin{abstract}
Due to major improvements in the management and therapy of patients with congenital adrenal hyperplasia owing to 21-hydroxylase deficiency (210HD) along childhood and adolescence, affected women are able to reach adulthood. Therefore, management throughout adult life became even more complex, leading to new challenges. Both the protracted use of corticosteroids (sometimes in supraphysiologic doses), and excess androgen (due to irregular treatment and/or inadequate dosage) may impair the quality of life and health outcomes in affected adult women, causing osteoporosis, metabolic disturbances with high cardiovascular risk, cosmetic damage, infertility, and psychosocial and psychosexual changes. However, long-term follow-up studies with $210 \mathrm{HD}$ adult women are still required. In this review, we discuss some important and controversial aspects of the follow-up of adult women with $210 \mathrm{HD}$, and recommend the use of a customized multi-disciplinary therapeutic approach while further studies with these patients do not provide distinct understanding and welldefined attitudes towards better quality of life. Arq Bras Endocrinol Metab. 2014;58(2):124-31
\end{abstract}

Keywords

21-hydroxylase deficiency; congenital adrenal hyperplasia; treatment; psychosocial and psychosexual aspects

\section{INTRODUÇÃO}

A deficiência de 21-hidroxilase (D21OH) é uma doença com padrão de herança autossômico recessivo resultante de mutações no gene CYP21A2, que codifica a enzima 21-hidroxilase (1); a D21OH corresponde a cerca de $90 \%$ dos casos de hiperplasia adrenal congênita (HAC). Como o defeito fundamental ocorre na síntese de cortisol, a elevação subsequente do ACTH determina hiperplasia e hiperestimulação dos córtices adrenais, produzindo um excesso de precursores esteroides e de hormônios sexuais, que não requerem 21-hidroxilação para sua síntese. Assim, a principal manifestação clínica dessa doença é a virilização precoce em meninos e meninas, além de aceleração do crescimento somático e fusão epifisária prematura.

A D21OH manifesta-se sob duas formas: clássica (FC) e não clássica (FNC), sendo a primeira subclassificada em formas perdedora de sal (PS) e virilizante 
simples (VS). Indivíduos com a forma VS apresentam virilização pré-natal com níveis elevados de andrógenos e precursores esteroides e produção preservada de aldosterona, enquanto aqueles com a forma PS são incapazes de sintetizar aldosterona em quantidade suficiente para manutenção do balanço hidroeletrolítico, constituindo a forma mais grave da doença (1). A FNC pode se manifestar desde a infância até a vida adulta com sinais e sintomas relacionados ao hiperandrogenismo (2). O grau de atividade residual da 21 -hidroxilase reflete a severidade das mutações no gene CYP21A2, existindo uma correlação genótipo-fenótipo significativa nos casos de D21OH $(1,3,4)$ (Figura 1$)$.

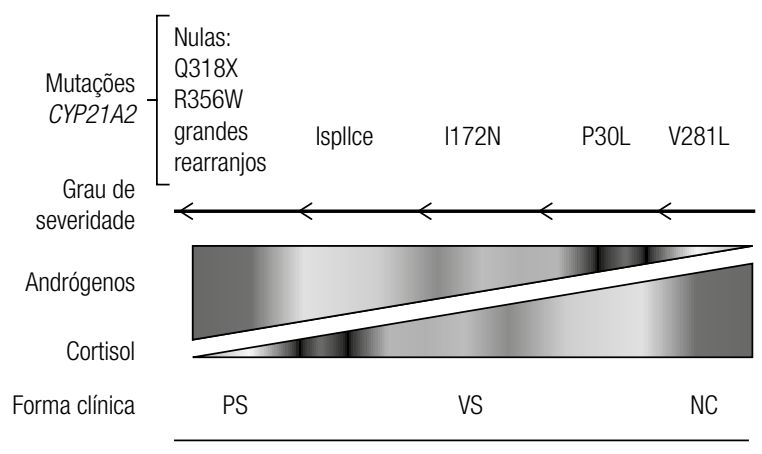

Figura 1. Correlação genótipo/fenótipo na D210H. Acima são indicadas as mutações mais frequentes no Brasil (ref. 3). As mutações nulas suprimem totalmente a atividade da enzima 21-hidroxilase, resultando em nenhuma produção de cortisol e maior nível de andrógenos em relação às outras formas (representado pela gradação de cores). Adaptado de Nordenstrom (4).

Estudo brasileiro de rastreamento neonatal estimou a incidência da FC da D21OH em 1:10.325 nascidos vivos (5). No entanto, apesar de não haver estudos de prevalência da FNC no Brasil, essa parece ser muito mais frequente, atingindo aproximadamente $0,1 \%$ da população geral (1), com maior incidência entre os judeus Askenazi (1:27 indivíduos). Esses dados comprovam que a FNC é a doença de padrão autossômico recessivo mais comum $(1,2)$.

Após a introdução do uso de glico- (GC) e mineralocorticoides (MC) no manejo das diversas formas da $\mathrm{D} 21 \mathrm{OH}$, a sobrevida dos pacientes, em especial dos portadores da FC (PS e VS), prolongou-se significativamente, e essa condição ganhou status de doença crônica (6). Como resultado, crianças com diagnóstico de $\mathrm{D} 21 \mathrm{OH}$ durante diferentes períodos da infância e adolescência passaram a ter seguimento clínico também durante a fase adulta. É a partir desse momento que as preocupações de longo prazo, tanto ligadas à doença em si como ao uso continuado de GC, tornam-se ainda mais desafiadoras. Consequências clínicas que eram temidas durante a infância (virilização, perda na estatura final e ganho de peso) somam-se, na vida adulta, a outras questões relevantes, como: complicações metabólicas (obesidade, diabetes e dislipidemia) com risco cardiovascular, osteoporose, infertilidade e comprometimento da sexualidade e da qualidade de vida. No entanto, são poucos os estudos que destacam a importância desses fatores na saúde da mulher adulta com D21OH (7-12).

Nesse artigo de revisão, abordamos alguns aspectos da dificuldade no manejo da $\mathrm{D} 21 \mathrm{OH}$ na mulher adulta, tanto na sua FC como FNC, enfatizando a necessidade de maior atenção e cuidado no manejo e realçando a importância da abordagem multidisciplinar no intuito de resolver os desafios envolvidos no seguimento dessas pacientes.

\section{DIAGNÓSTICO NA MULHER ADULTA}

Enquanto mulheres adultas com a FC já carregam o diagnóstico de $\mathrm{D} 21 \mathrm{OH}$ desde a infância, a maioria daquelas com a FNC só será identificada e investigada se e quando apresentar manifestações clínicas de hiperandrogenismo (incluindo irregularidade menstrual) e/ou infertilidade.

Em decorrência da excessiva produção de andrógenos adrenais, esses são parcialmente aromatizados em estrógenos, possibilitando um aumento na sensibilidade hipofisária do LH ao GnRH e consequente perda de ciclicidade da secreção de gonadotrofinas. Esse mecanismo leva à disfunção ovariana e à anovulação, mimetizando a síndrome dos ovários policísticos (SOP) ("estado SOP-like") $(13,14)$. Séries que determinam a frequência da FNC em mulheres hiperandrogênicas mostram estimativas entre 1,2 e $20 \%(2,15-18)$.

$\mathrm{O}$ marcador diagnóstico da $\mathrm{D} 21 \mathrm{OH}$ é a 17-hidroxiprogesterona sérica (17OHP), precursor imediato da 21-hidroxilase. Para contornar as flutuações dos seus valores, a coleta para $17 \mathrm{OHP}$ deve ser feita na fase folicular precoce, em torno das 8 horas da manhã $(19,20)$. Níveis basais muito elevados da 17OHP (em geral 20 a 100 vezes o valor máximo de referência) são diagnósticos para a FC da $\mathrm{D} 2 \mathrm{lOH}$, porém valores basais pouco elevados e mesmo normais podem ocorrer na FNC. Da mesma maneira, mulheres com diagnóstico clínico de hiperandrogenismo não relacionado à $\mathrm{D} 21 \mathrm{OH}$ podem 
ter níveis basais de 17OHP pouco elevados (21). Para essa diferenciação, níveis de 17OHP dosados 60 minutos após a administração intravenosa de ACTH sintético (cosintropina) mostram-se críticos; após estímulo, o valor de corte da $17 \mathrm{OHP}$ tradicionalmente utilizado no diagnóstico da FNC é de 1.000 ng/dL $(2,19,20)$ (Figura 2). Contudo, valores abaixo de $1.000 \mathrm{ng} / \mathrm{dL}$ não discriminam heterozigotos para $\mathrm{D} 21 \mathrm{OH}$ de indivíduos genotipicamente normais.

Embora a dosagem de 21-deoxicortisol (21DF, esteroide resultante da $11 \beta$-hidroxilação da $17 \mathrm{OHP}$ ) possa ser um melhor marcador para a identificação de heterozigotos na população normal $(10,22)$, a genotipagem da CYP21A2 ainda é o padrão-ouro para
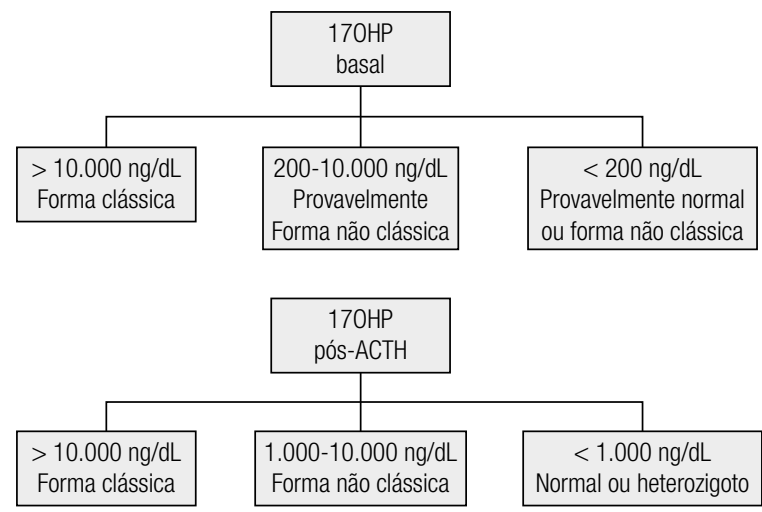

Figura 2. Algoritmo diagnóstico da $\mathrm{D} 210 \mathrm{H}$, de acordo com as diretrizes da Endocrine Society de 2010. Adaptada de Speiser e cols. (19). essa diferenciação. Uma vez que níveis estimulados de 17OHP ainda não estejam definitivamente estabelecidos para o diagnóstico da FNC da D21OH, Bachega e cols. propõem um valor de corte de $1.700 \mathrm{ng} / \mathrm{dL}$, bastante concordante com a genotipagem da CYP2IA2 (23). Adicionalmente, em estudo realizado na Unifesp, os valores de $21 \mathrm{DF}$ compatíveis com o diagnóstico da FNC foram de $\geq 40 \mathrm{ng} / \mathrm{dL}$ e $\geq 300 \mathrm{ng} / \mathrm{dL}$, respectivamente no basal e após estímulo com $\mathrm{ACTH}$, não havendo sobreposição dos valores com heterozigotos ou indivíduos genotipicamente normais (22) (Figura 3).

\section{CORTICOTERAPIA}

Tanto na criança como na mulher adulta, o objetivo do tratamento é administrar a dose mínima efetiva de GC capaz de bloquear o aumento de ACTH e, consequentemente, normalizar a concentração de andrógenos. $\mathrm{Na}$ FC, a terapia deve ser continuada na vida adulta, mas na FNC sugere-se que o tratamento deva ser feito apenas nas mulheres com manifestações de hiperandrogenismo significativo (incluindo avanço na idade óssea) e/ou infertilidade $(19,20)$.

$\mathrm{Na}$ infância e adolescência, o GC de eleição no tratamento da $\mathrm{D} 2 \mathrm{lOH}$ é a hidrocortisona, em doses de 10 a $15 \mathrm{mg} / \mathrm{m}^{2} \mathrm{SC} /$ dia $(19,20)$, mas na mulher adulta com $\mathrm{D} 21 \mathrm{OH}$ não há estudos controlados que definam a melhor opção de uso de GC. Contudo, segundo

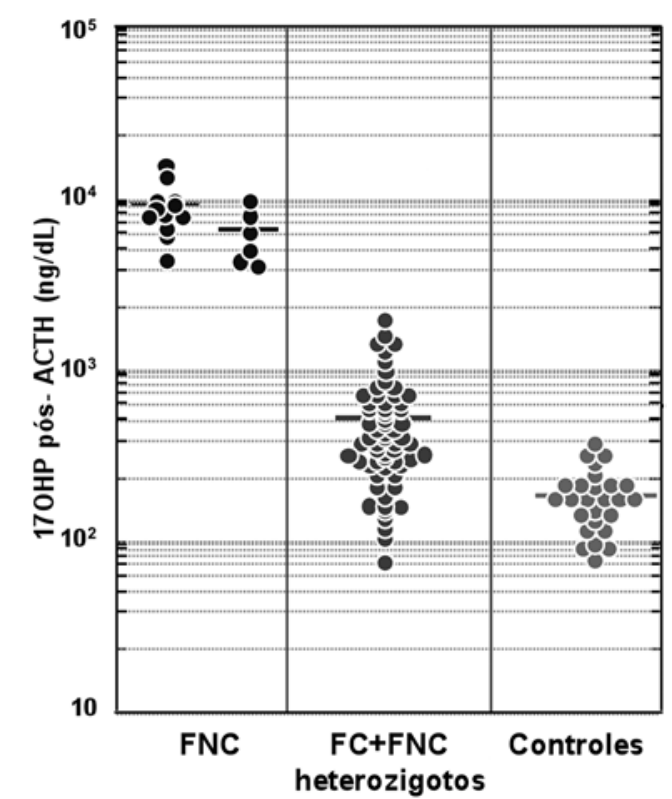

Figura 3. Valores de 21DF e 170HP após ACTH na D210H: pacientes com FNC sem tratamento prévio (esquerda), previamente tratados (direita), heterozigotos para FC e FNC e indivíduos controles genotipicamente normais. Adaptado de Costa-Barbosa e cols. (22). 
as recomendações da Endocrine Society e da CARES Foundation, os GC sintéticos de longa duração são preferíveis no seguimento de todas as formas de $\mathrm{D} 2 \mathrm{lOH}$ $(19,20)$. O uso de dexametasona na dose de $0,25 \mathrm{mg} /$ dia na FNC pode ser efetivo em controlar a acne e a irregularidade menstrual num prazo de 30 meses, segundo experiência clínica de New (2). No entanto, a "supressão" androgênica pelo uso da dexametasona (implicando doses excessivas) associa-se ao surgimento de efeitos colaterais, em especial aqueles relacionados à resistência insulínica (24), mas apresenta caráter individual. As doses iniciais de dexametasona variam em torno de 0,25 a $0,375 \mathrm{mg} /$ dia, mas algumas pacientes se beneficiam com doses de até $0,5 \mathrm{mg} /$ dia $(2,25)$.

Outra opção bem aceita de GC é a prednisolona, que pode ser utilizada em doses baixas para controle das manifestações decorrentes da D21OH. O Ambulatório de Doenças Adrenais da Unifesp e o Serviço de Endocrinologia Pediátrica da Santa Casa de São Paulo têm experiência com o uso de prednisolona em crianças com a FC na dose de 1,5 a $3 \mathrm{mg} / \mathrm{m}^{2} \mathrm{SC} /$ dia, administrada em uma ou eventualmente duas tomadas $(26,27)$. Nas mulheres adultas, bons resultados são obtidos com doses de 2 a $3 \mathrm{mg} /$ dia, divididos em uma ou duas doses diárias. Para tanto, temos preferido o emprego de preparações líquidas de prednisolona (fornecidas em concentrações de 1 e $3 \mathrm{mg} / \mathrm{mL}$ ), dada a rigidez das formulações comerciais em comprimidos no Brasil (apenas comprimidos de 5 e $20 \mathrm{mg}$ ) $(7,25)$. O controle de parâmetros clínicos, como a melhora das manifestações de hiperandrogenismo, regularização dos ciclos menstruais e ovulação, é o objetivo precípuo do tratamento dessas pacientes, independente dos resultados laboratoriais. Isso se deve ao fato de que a normalização da concentração sérica de $17 \mathrm{OHP}$ como objetivo laboratorial (ainda vista como ideal por muitos) leva sistematicamente o paciente a apresentar manifestações cushingoides.

Quanto aos MC, adultos com a FC necessitam de doses menores de fludrocortisona $(50-100 \mathrm{mcg} / \mathrm{dia})$ comparadas às doses usadas nos primeiros anos de vida e infância $(100-200 \mathrm{mcg} /$ dia $)(6,7,19,28)$. Não se sabe ao certo o mecanismo envolvido na melhora da sensibilidade com a idade, mas parece estar relacionado com a redução da resistência renal à ação dos $\mathrm{MC}$, que ocorre após os primeiros anos de vida (29). A dose ideal de fludrocortisona é aquela que mantém a atividade plasmática de renina (APR) na faixa normal, de modo que controle a tendência à perda salina, sem causar hiper- tensão ou hipocalemia. Por isso, as dosagens da APR e de sódio e potássio tornam-se imperativas para a decisão de ajustes terapêuticos nas pacientes com a FC. De modo geral, mulheres adultas com a FNC não requerem tratamento com MC (25). Estudo recente demonstrou uma pobre correlação entre o genótipo e o tratamento de mulheres adultas com diversas formas de $\mathrm{D} 21 \mathrm{OH}$, mas nenhuma correlação entre o genótipo e o estado de saúde destas (30).

\section{TRATAMENTO COSMÉTICO}

Como já referido, mulheres com D21OH apresentam um quadro secundário de SOP decorrente do excesso de andrógenos adrenais. Caso a mulher não queira engravidar, ou naquelas com a FNC cujos sinais de hiperandrogenismo sejam mais leves, o tratamento pode ser feito de maneira similar ao da SOP (25). O uso de contraceptivos orais (elevando a SHBG e reduzindo os níveis de testosterona livre) e/ou espironolactona (pelo seu efeito antiandrogênico ao nível do receptor de andrógenos) pode ser uma boa opção terapêutica. No entanto, deve-se salientar que essas possibilidades são apenas paliativas, procurando atenuar as manifestações do hiperandrogenismo. Outras opções mecânicas como depilação, eletrólise, laser e cremes tópicos (eflornitina) podem ser utilizadas, isoladas ou em combinação, para a melhora temporária do hirsutismo.

\section{ADRENALECTOMIA BILATERAL}

Embora possa ser opção de tratamento definitivo em mulheres com a FC e infertilidade $(31,32)$, a adrenalectomia bilateral deve ser indicada apenas excepcionalmente, em especial naquelas pacientes com boa aderência, mas refratárias à terapia medicamentosa $(19,20)$. O uso eventual desse procedimento deve-se ao maior risco cirúrgico e anestésico, além do importante risco potencial de insuficiência adrenal total (6).

\section{SEXUALIDADE}

A literatura relata que mulheres com D21OH têm menos relacionamentos (sexuais e amorosos) e em menor frequência do que mulheres da população geral $(33,34)$. Algumas pacientes, em especial aquelas com a forma PS, evitavam se relacionar por temer a rejeição dos parceiros $(34,35)$; outras referiam se sentir menos atraentes e femininas, julgando-se incapazes de seduzir 
um parceiro, demonstrando grande insegurança e baixa autoestima.

Outros estudos (36-40) descrevem que mulheres com a FC iniciam seu relacionamento sexual mais tardiamente (em média dois anos), costumam ser menos ativas sexualmente e ter menos filhos. Apesar dos avanços cirúrgicos, algumas mulheres adultas ainda se queixam de dispareunia e dificuldade para atingir o orgasmo $(35,36)$. Os procedimentos de dilatação, muito usados no passado, ainda são descritos pelas pacientes como doloridos e vexatórios (41).

Quanto à orientação sexual, a maioria reporta orientação heterossexual, mas a percentagem de casos de homossexualismo e bissexualismo é mais elevada do que na população geral, ocorrendo em especial nas pacientes mais severamente afetadas pela doença (39). Esse é um dos aspectos que coloca em discussão a identidade sexual de pacientes com a forma severa da $\mathrm{D} 21 \mathrm{OH}$, levando alguns autores a julgarem oportuno criar esses pacientes no sexo masculino (42).

\section{FERTILIDADE}

Estudos demonstram que a fertilidade e a fecundidade encontram-se reduzidas nas mulheres com a FC da $\mathrm{D} 21 \mathrm{OH}$ (43-46). Existem vários fatores que podem explicar essa situação: (a) alterações anatômicas do introito vaginal decorrentes da virilização genital e de sequelas da correção cirúrgica $(11,43)$, (b) manutenção da hipersecreção androgênica com consequente anovulação crônica $(10,14)$ e (c) distúrbios de imagem corporal, levando a dificuldades de relacionamentos (45). Outro aspecto relevante relaciona-se aos níveis excessivos de 17OHP e especialmente de progesterona, que podem interferir no processo de implantação do embrião (47); em geral, valores de progesterona mantidos abaixo de $60 \mathrm{ng} / \mathrm{dL}$ favorecem a fertilidade nessas pacientes (45).

No entanto, mesmo com a fertilidade reduzida, as taxas de gestação em pacientes com a FC são apenas um pouco menores se comparadas à população geral (6). Estudo recente demonstrou que apenas 23 de 106 mulheres $(21,7 \%)$ com a FC tentaram ativamente engravidar (45). Dessas, 21 (91,3\%) obtiveram sucesso, caracterizando, assim, uma taxa normal de gestação, se comparada com a taxa de sucesso de $95 \%$ da população geral. Por outro lado, estudo multicêntrico do Reino Unido, baseado em dados de questionários, revelou uma taxa de sucesso menor, de $54 \%$ (7).
Em contraste com a FC, a fertilidade na FNC encontra-se menos afetada e pode estar relacionada principalmente ao desbalanço hormonal causado pelo hiperandrogenismo adrenal. Como já referido, mulheres com a FNC apresentam disfunção ovariana e anovulação, caracterizando um "estado SOP-like". No entanto, estudos mostram que a anovulação crônica e a fertilidade geralmente podem ser corrigidas pelo uso apropriado de GC. Adicionalmente, relatos recentes demonstraram que o uso de GC nessas pacientes está associado a uma menor taxa de abortamento $(10,48)$, enfatizando a importância da corticoterapia nas mulheres com a FNC que tenham a fertilidade comprometida.

Quando engravidar, a paciente deverá ser acompanhada conjuntamente pelo obstetra e o endocrinologista. A terapia de escolha para a gestante é a hidrocortisona, que sofre degradação pela enzima $11 \beta$ hidroxiesteroide-desidrogenase do tipo II (11ßHSDII), não ultrapassando a barreira placentária. $\mathrm{O}$ uso de GC e MC deverá ser iniciado na mesma dose do período pré-gestacional. Entretanto, pode haver necessidade do aumento da dose dessas medicações, devido à elevação dos níveis de proteínas ligadoras do cortisol (CBG) durante a gestação.

Fato importante, caso a genotipagem da paciente mostrar pelo menos um alelo afetado com mutação grave (àquelas relacionadas com FC) e o parceiro for heterozigoto também para essas mutações, a prole poder apresentar a FC com consequente virilização genital no feto do sexo feminino. Nessa circunstância, habitualmente opta-se pelo uso de dexametasona (não é degradada pela $11 \beta H S D-I I$ placentária) com objetivo de reduzir a exposição pré-natal ao excesso androgênico. No entanto, devido a um risco maior de teratogenicidade, e por não haver estudos suficientes em meninas que se beneficiaram dessa terapia, o último Consenso da Endocrine Society $(19,20)$ sugere que o uso de dexametasona durante a gestação deva ser reavaliado, sendo restrito a protocolos de pesquisa em ambiente acadêmico.

Adicionalmente, deve-se atentar aos efeitos colaterais do uso prolongado de GC e mesmo à insuficiência adrenal durante o trabalho de parto ou outros períodos de estresse endógeno, especialmente na forma PS.

\section{ASPECTOS PSICOSSOCIAIS E PSICOSSEXUAIS}

O tratamento de mulheres com FC e FNC exige uma atenção especial aos aspectos psicossociais e psicossexuais. Esses aspectos dizem respeito a: (a) avaliação da 
atribuição de gênero; (b) períodos das intervenções médicas; (c) melhor momento para se realizar a cirurgia corretiva, bem como seus resultados e consequências; (d) a sexualidade e a orientação sexual e (e) a maneira como as pacientes deverão receber as informações acerca do seu diagnóstico - a gestão das informações (49-51). A literatura é unânime em afirmar que a qualidade de vida das mulheres com $\mathrm{D} 21 \mathrm{OH}$ depende diretamente da maneira como esse manejo é ou foi feito $(19,30,33,34,52)$.

Sabe-se que o diagnóstico da $\mathrm{D} 21 \mathrm{OH}$ tem um impacto direto sobre a vida dessas mulheres e que, por esse motivo, os centros especializados nos seus cuidados devem estar atentos aos aspectos já mencionados desde a infância até a vida adulta. Na história das Desordens do Desenvolvimento Sexual (DDS) (53), relatos de casos mal conduzidos demonstraram consequências drásticas no desenvolvimento da criança e, posteriormente, problemas na vida adulta, tais como: desejo de mudar de sexo quando adolescente e/ou adulto, depressões, fobias sociais, inibições no trato social, transtornos de ansiedade e tentativas de suicídio $(33,49,51,54)$.

A avaliação das pacientes na vida adulta requer um cuidado individualizado e especializado, principalmente no que concerne à gestão da informação, sendo esse um momento oportuno para compreensão da história da doença, seu diagnóstico e tratamento $(49,51,55)$. Estudos realizados por diferentes grupos $(49,51,55)$ evidenciaram que existe uma disparidade, entre o médico e o público leigo, na compreensão da doença e seu tratamento. Por esse motivo, sugere-se que os profissionais da área da saúde mental façam atendimentos de avaliação desses pacientes com o propósito de verificar o quanto estes assimilaram da doença e seu tratamento, favorecendo e facilitando a interlocução entre o paciente e a equipe médica.

Com o mesmo intuito de apontar a carência nos estudos de mulheres adultas com D21OH e suas consequências no manejo dos aspectos psicossociais, Jordan-Young (56) menciona, em sua revisão sobre as influências dos hormônios no cérebro, que as publicações têm negligenciado importantes aspectos: a) os efeitos fisiopatológicos da HAC pós-natal, b) as intervenções constantes e as consultas médicas descritas por algumas pacientes como sendo traumáticas, c) os efeitos diretos da virilização genital sobre a sexualidade e d) a avaliação de gênero e a sexualidade da paciente. Tais tópicos contribuem para a discussão entre as interações complexas das variáveis biológicas, sociais e psicológicas que afetam os comportamentos atípicos de algumas mulheres com D21OH e evidenciam que a explicação convencional do "imprint" cerebral no período crítico é insuficiente para abordar toda a complexidade dessas questões. Segundo a autora, os estudos que defendem essa teoria se concentram estritamente nas exposições hormonais no início da vida e excluem praticamente todas as outras variáveis que afetam o desenvolvimento (56).

Para algumas mulheres, as manifestações hormonais da $\mathrm{D} 21 \mathrm{OH}$ permanecem um enigma até a vida adulta, com algumas delas desconhecendo a própria condição. Muitas vezes, as pacientes realizam um pacto de silêncio com seus pais, acreditando que assim poderão evitar mais sofrimentos para ambas as partes (55). $\mathrm{Na}$ idade adulta, no entanto, o médico passa a ser um importante interlocutor no acolhimento e direcionamento dessas questões. Desse modo, os especialistas em atendimento de pacientes adultas devem estar preparados para responder às perguntas que tais pacientes poderão fazer, tendo em mente que essa dificuldade em assimilar a própria doença pode trazer consequências diretas no que se refere ao desenvolvimento psíquico e social. Cabe ressaltar que esse manejo deve ser refinado e cauteloso para adequar as informações ao nível cognitivo da paciente, bem como para discriminar o melhor momento para revelar determinadas informações.

\section{OUTRAS CONSIDERAÇÕES}

Apesar de estudos de densidade mineral óssea (DMO) em mulheres adultas acima de 30 anos e naquelas pós-menopausadas evidenciarem um aumento na prevalência de osteopenia e osteoporose quando comparado com indivíduos saudáveis $(7,57,58)$, não há dados suficientes para orientar o emprego regular da DMO fora do ambiente acadêmico. Além disso, essas mulheres têm maior prevalência de obesidade, resistência à insulina, dislipidemia e hipertensão (24). Esses fatores em conjunto traduzem a síndrome metabólica, com consequente risco cardiovascular aumentado $(7,59,60)$. No entanto, ainda não foi demonstrada maior morbidade ou mortalidade cardiovascular ou risco de diabetes tipo 2 nessas mulheres $(9)$.

\section{CONCLUSÕES}

Em função da carência de dados concretos sobre a saúde da mulher adulta com $\mathrm{D} 21 \mathrm{OH}$, recomendamos 
que o manejo deva ser individualizado, mas prestando atenção nos conceitos atuais relacionados à complexidade dessa doença. Sugere-se que o melhor parâmetro de tratamento para essas pacientes seja uma avaliação clínica criteriosa, somada aos cuidados psicossociais que devem ser tratados em centros especializados em HAC, garantindo, assim, uma melhor qualidade de vida para essas pacientes.

Declaração: os autores declaram não haver conflitos de interesse científico neste estudo.

\section{REFERÊNCIAS}

1. White PC, Speiser PW. Congenital adrenal hyperplasia due to 21-hydroxylase deficiency. Endocr Rev. 2000;21(3):245-91.

2. New MI.Extensive clinical experience: nonclassical 21-hydroxylase deficiency. J Clin Endocrinol Metab. 2006;91(11):4205-14.

3. BachegaTA, Billerbeck AE, Parente EB, Lemos-Marini SH, Baptista MT, Mello MP, et al. [Multicentric study of Brazilian patients with 21-hydroxylase deficiency: a genotype-phenotype correlation]. Arq Bras Endocrinol Metabol. 2004;48(5):697-704.

4. Nordenstrom A. Adult women with 21-hydroxylase deficient congenital adrenal hyperplasia, surgical and psychological aspects. Curr Opin Pediatr. 2011;23(4):436-42.

5. Silveira EL, dos Santos EP, Bachega TA, van der Linden Nader I, Gross JL, Elnecave RH. The actual incidence of congenital adrenal hyperplasia in Brazil may not be as high as inferred--an estimate based on a public neonatal screening program in the state of Goias. J Pediatr Endocrinol Metab. 2008;21(5):455-60.

6. Reisch N, Arlt W, Krone N. Health problems in congenital adrenal hyperplasia due to 21-hydroxylase deficiency. Horm Res Paediatr. 2011;76(2):73-85.

7. Arlt W, Willis DS, Wild SH, Krone N, Doherty EJ, Hahner S, et al. Health status of adults with congenital adrenal hyperplasia: a cohort study of 203 patients. J Clin Endocrinol Metab. 2010;95(11):5110-21.

8. Bachelot A, Plu-Bureau G, Thibaud E, Laborde K, Pinto G, Samara $D$, et al. Long-term outcome of patients with congenital adrenal hyperplasia due to 21-hydroxylase deficiency. Horm Res. 2007;67(6):268-76.

9. Falhammar H, Filipsson H, Holmdahl G, Janson PO, Nordenskjold A, Hagenfeldt $K$, et al. Metabolic profile and body composition in adult women with congenital adrenal hyperplasia due to 21-hydroxylase deficiency. J Clin Endocrinol Metab. 2007;92(1):110-6.

10. Bidet $M$, Bellanne-Chantelot C, Galand-Portier MB, Tardy V, Billaud L, Laborde K, et al. Clinical and molecular characterization of a cohort of 161 unrelated women with nonclassical congenital adrenal hyperplasia due to 21-hydroxylase deficiency and 330 family members. J Clin Endocrinol Metab. 2009;94(5):1570-8.

11. Premawardhana LD, Hughes IA, Read GF, Scanlon MF. Longer term outcome in females with congenital adrenal hyperplasia (CAH): the Cardiff experience. Clin Endocrinol (Oxf). 1997;46(3):327-32.

12. Cabrera MS, Vogiatzi MG, New MI. Long term outcome in adult males with classic congenital adrenal hyperplasia. J Clin Endocrinol Metab. 2001;86(7):3070-8.

13. Bidet $M$, Bellanne-Chantelot $C$, Galand-Portier MB, Golmard $\mathrm{JL}$, Tardy V, Morel Y, et al. Fertility in women with nonclassical congenital adrenal hyperplasia due to 21-hydroxylase deficiency. J Clin Endocrinol Metab. 2010.;95(3):1182-90.
14. Feldman S, Billaud L,Thalabard JC, Raux-Demay MC, Mowszowicz I, Kuttenn $F$, et al. Fertility in women with late-onset adrenal hyperplasia due to 21-hydroxylase deficiency. J Clin Endocrinol Metab. 1992;74(3):635-9.

15. Chetkowski RJ, DeFazio J, Shamonki I, Judd HL, Chang RJ. The incidence of late-onset congenital adrenal hyperplasia due to 21-hydroxylase deficiency among hirsute women. J Clin Endocrinol Metab. 1984;58(4):595-8.

16. Kuttenn F, Couillin P, Girard F, Billaud L, Vincens M, Boucekkine C, et al. Late-onset adrenal hyperplasia in hirsutism. N Engl J Med. 1985;313(4):224-31.

17. Sahin $Y$, Kelestimur $F$. The frequency of late-onset 21-hydroxylase and 11 beta-hydroxylase deficiency in women with polycystic ovary syndrome. Eur J Endocrinol. 1997;137(6):670-4.

18. Azziz R, Sanchez LA, Knochenhauer ES, Moran C, Lazenby J, Stephens KC, et al. Androgen excess in women: experience with over 1000 consecutive patients. J Clin Endocrinol Metab. 2004;89(2):453-62.

19. Speiser PW, Azziz R, Baskin LS, Ghizzoni L, HensleTW, Merke DP, et al. Congenital adrenal hyperplasia due to steroid 21-hydroxylase deficiency: an Endocrine Society clinical practice guideline. J Clin Endocrinol Metab. 2010;95(9):4133-60.

20. Auchus RJ, Witchel SF, Leight KR, Aisenberg J, Azziz R, Bachega TA, et al. Guidelines for the Development of Comprehensive Care Centers for Congenital Adrenal Hyperplasia: Guidance from the CARES Foundation Initiative. Int J Pediatr Endocrinol. 2010;2010:275213.

21. Azziz R, Hincapie LA, Knochenhauer ES, Dewailly D, Fox L, Boots LR. Screening for 21-hydroxylase-deficient nonclassic adrenal hyperplasia among hyperandrogenic women: a prospective study. Fertil Steril. 1999;72(5):915-25.

22. Costa-Barbosa FA, Tonetto-Fernandes VF, Carvalho VM, Nakamura $\mathrm{OH}$, Moura V, Bachega TA, et al. Superior discriminating value of ACTH-stimulated serum 21-deoxycortisol in identifying heterozygote carriers for 21-hydroxylase deficiency. Clin Endocrinol (Oxf). 2010;73(6):700-6.

23. Bachega TA, Billerbeck AE, Marcondes JA, Madureira G, Arnhold IJ, Mendonca BB. Influence of different genotypes on 17-hydroxyprogesterone levels in patients with nonclassical congenital adrenal hyperplasia due to 21-hydroxylase deficiency. Clin Endocrinol (Oxf). 2000;52(5):601-7.

24. Han TS, Stimson RH, Rees DA, Krone N, Willis DS, Conway GS, et al. Glucocorticoid treatment regimen and health outcomes in adults with congenital adrenal hyperplasia. Clin Endocrinol (Oxf). 2013;78(2):197-203.

25. Auchus RJ, Arlt W. Approach to the patient: the adult with congenital adrenal hyperplasia. J Clin Endocrinol Metab. 2013;98(7):2645-55.

26. Caldato MC, Fernandes VT, Kater CE. One-year clinical evaluation of single morning dose prednisolone therapy for 21-hydroxylase deficiency. Arq Bras Endocrinol Metabol. 2004;48(5):705-12.

27. Leite FM, Longui CA, Kochi C, Faria CD, Borghi M, Calliari LE, et al. [Comparative study of prednisolone versus hydrocortisone acetate for treatment of patients with the classic congenital adrenal hyperplasia due to 21-hydroxylase deficiency]. Arq Bras Endocrinol Metabol. 2008;52(1):101-8.

28. Gomes LG, Madureira G, Mendonca BB, Bachega TA. Mineralocorticoid replacement during infancy for salt wasting congenital adrenal hyperplasia due to 21 -hydroxylase deficiency. Clinics (Sao Paulo). 2013;68(2):147-52.

29. Martinerie L, Pussard E, Foix-L'Helias L, Petit F, Cosson C, Boileau $P$, et al. Physiological partial aldosterone resistance in human newborns. Pediatr Res. 2009;66(3):323-8.

30. Krone N, Rose IT, Willis DS, Hodson J, Wild SH, Doherty EJ, et al. Genotype-phenotype correlation in 153 adult patients with 
congenital adrenal hyperplasia due to 21-hydroxylase deficiency: analysis of the United Kingdom Congenital adrenal Hyperplasia Adult Study Executive (CaHASE) cohort. J Clin Endocrinol Metab. 2013;98(2):E346-54.

31. Van Wyk JJ, Ritzen EM. The role of bilateral adrenalectomy in the treatment of congenital adrenal hyperplasia. J Clin Endocrinol Metab. 2003;88(7):2993-8.

32. Ogilvie CM, Rumsby G, Kurzawinski T, Conway GS. Outcome of bilateral adrenalectomy in congenital adrenal hyperplasia: one unit's experience. Eur J Endocrinol. 2006;154(3):405-8.

33. Malouf MA, Inman AG, Carr AG, Franco J, Brooks LM. Healthrelated quality of life, mental health and psychotherapeutic considerations for women diagnosed with a disorder of sexual development: congenital adrenal hyperplasia. Int J Pediatr Endocrinol. 2010;2010:253465.

34. Arlt W, Krone N. Adult consequences of congenital adrenal hyperplasia. Horm Res. 2007;68 Suppl 5:158-64.

35. Nordenstrom A, Frisen L, Falhammar H, Filipsson $\mathrm{H}$, Holmdahl $G$, Janson PO, et al. Sexual function and surgical outcome in women with congenital adrenal hyperplasia due to CYP21A2 deficiency: clinical perspective and the patients' perception. J Clin Endocrinol Metab. 2010;95(8):3633-40.

36. Fagerholm R, Santtila P, Miettinen PJ, Mattila A, Rintala R, Taskinen S. Sexual function and attitudes toward surgery after feminizing genitoplasty. J Urol. 2011;185(5):1900-4.

37. Nermoen I, Husebye ES, Svartberg J, Lovas K. Subjective health status in men and women with congenital adrenal hyperplasia: a population-based survey in Norway. Eur $\mathrm{J}$ Endocrinol. 2010;163(3):453-9.

38. Hagenfeldt $\mathrm{K}$, Janson PO, Holmdahl G, Falhammar H, Filipsson $H$, Frisen $L$, et al. Fertility and pregnancy outcome in women with congenital adrenal hyperplasia due to 21-hydroxylase deficiency. Hum Reprod. 2008;23(7):1607-13.

39. Meyer-Bahlburg HF, Dolezal C, Baker SW, New MI. Sexual orientation in women with classical or non-classical congenital adrenal hyperplasia as a function of degree of prenatal androgen excess. Arch Sex Behav. 2008;37(1):85-99.

40. Frisen L, Nordenstrom A, Falhammar H, Filipsson H, Holmdahl G, Janson PO, et al. Gender role behavior, sexuality, and psychosocial adaptation in women with congenital adrenal hyperplasia due to CYP21A2 deficiency. J Clin Endocrinol Metab. 2009;94(9):3432-9.

41. Telles-Silveira $M$, Tonetto-Fernandes VF, Schiller $P$, Kater CE. [Congenital adrenal hyperplasia: a qualitative study on sex definition and redesignation, dilation, surgery and psychological support (part II)]. Arq Bras Endocrinol Metabol. 2009;53(9):1125-36.

42. Lee PA, Houk CP, Husmann DA. Should male gender assignment be considered in the markedly virilized patient With $46, X X$ and congenital adrenal hyperplasia? J Urol. 2010;184(4 Suppl):1786-92.

43. Mulaikal RM, Migeon CJ, Rock JA. Fertility rates in female patients with congenital adrenal hyperplasia due to 21-hydroxylase deficiency. N Engl J Med. 1987;316(4):178-82.

44. Pardini DP, Kater CE, Vasserman J, Baracat EC, Chacra AR, de Lima GR. [Congenital adrenal hyperplasia and pregnancy]. Rev Paul Med. 1989;107(2):88-92.

45. Casteras A, De Silva P, Rumsby G, Conway GS. Reassessing fecundity in women with classical congenital adrenal hyperplasia
$(\mathrm{CAH})$ : normal pregnancy rate but reduced fertility rate. Clin Endocrinol (Oxf). 2009;70(6):833-7.

46. Reisch N, Flade L, Scherr M, Rottenkolber M, Pedrosa Gil F, Bidlingmaier $\mathrm{M}$, et al. High prevalence of reduced fecundity in men with congenital adrenal hyperplasia. J Clin Endocrinol Metab. 2009;94(5):1665-70.

47. Holmes-Walker DJ, Conway GS, Honour JW, Rumsby G, Jacobs HS. Menstrual disturbance and hypersecretion of progesterone in women with congenital adrenal hyperplasia due to 21-hydroxylase deficiency. Clin Endocrinol (Oxf). 1995;43(3):291-6.

48. Moran C, Azziz R, Weintrob N, Witchel SF, Rohmer V, Dewailly $D$, et al. Reproductive outcome of women with 21-hydroxylasedeficient nonclassic adrenal hyperplasia. J Clin Endocrinol Metab. 2006;91(9):3451-6.

49. Cohen-Kettenis PT. Psychosocial and psychosexual aspects of disorders of sex development. Best Pract Res Clin Endocrinol Metab. 2010;24(2):325-34.

50. Houk CP, Lee PA. Update on disorders of sex development. Curr Opin Endocrinol Diabetes Obes. 2012;19(1):28-32.

51. Meyer-Bahlburg HF. Brain development and cognitive, psychosocial, and psychiatric functioning in classical 21-hydroxylase deficiency. Endocr Dev. 2011.20:88-95.

52. Liao LM, Green H, Creighton SM, Crouch NS, Conway GS. Service users' experiences of obtaining and giving information about disorders of sex development. BJOG. 2010;117(2):193-9.

53. Lee PA, Houk CP, Ahmed SF, Hughes IA. Consensus statement on management of intersex disorders. International Consensus Conference on Intersex. Pediatrics. 2006;118(2):e488-500.

54. Kleinemeier $E$, Jurgensen M, Lux A, Widenka PM, Thyen $U$. Psychological adjustment and sexual development of adolescents with disorders of sex development. J Adolesc Health. 2010;47(5):463-71.

55. Telles-Silveira M, Tonetto-Fernandes VF, Schiller $P$, Kater CE. [Congenital adrenal hyperplasia: a qualitative study on disease and treatment, doubts, anguishes and relationships (part I)]. Arq Bras Endocrinol Metabol. 2009;53(9):1112-24.

56. Jordan-Young RM. Hormones, context, and "brain gender": a review of evidence from congenital adrenal hyperplasia. Soc Sci Med. 2012;74(11):1738-44.

57. King JA, Wisniewski AB, Bankowski BJ, Carson KA, Zacur HA, Migeon CJ. Long-term corticosteroid replacement and bone mineral density in adult women with classical congenital adrenal hyperplasia. J Clin Endocrinol Metab. 2006;91(3):865-9.

58. Falhammar H, Filipsson $\mathrm{H}$, Holmdahl G, Janson PO, Nordenskjold A, Hagenfeldt $K$, et al. Fractures and bone mineral density in adult women with 21-hydroxylase deficiency. J Clin Endocrinol Metab. 2007;92(12):4643-9.

59. Mooij CF, Kroese JM, Claahsen-van der Grinten HL, Tack CJ, Hermus AR. Unfavourable trends in cardiovascular and metabolic risk in paediatric and adult patients with congenital adrenal hyperplasia? Clin Endocrinol (Oxf). 2010;73(2):137-46.

60. Zimmermann A, Grigorescu-Sido P, AlKhzouz C, Patberg K, Bucerzan S, Schulze E, et al. Alterations in lipid and carbohydrate metabolism in patients with classic congenital adrenal hyperplasia due to 21-hydroxylase deficiency. Horm Res Paediatr. $2010 ; 74(1): 41-9$. 